\title{
CARACTERIZAÇÃO DA MICROBIOTA CUTÂNEA DE Aplastodiscus leucopygius DO PARQUE MUNICIPAL NATURAL AUGUSTO RUSCHI
}

\author{
Larissa Nolasco Fernandes ${ }^{1}$ \\ Mariana Maykot Serafim ${ }^{2}$ \\ Claudineia Aparecida da Silva Araujo $^{3}$ \\ Jonatas Alves Ferreira Neto ${ }^{4}$ \\ Matheus de Toledo Moroti ${ }^{5}$ \\ Cristina Pacheco Soares ${ }^{6}$ \\ Flavia Villaça Morais ${ }^{7}$
}

Resumo: A Aplastodiscus leucopygius é um anfíbio da ordem Anura pertencente à família Hylidae, espécie endêmica do Brasil, encontrada em áreas de Mata Atlântica do planalto e serras do Mar e da Mantiqueira, nos Estados de São Paulo e Rio de Janeiro. O presente estudo teve como objetivo analisar e caracterizar a microbiota de A. leucopygius encontrados no Parque Municipal Natural Augusto Ruschi, Unidade de Conservação de Proteção Integral da cidade de São José do Campos no estado de São Paulo. Para isso foram coletadas e analisadas amostras biológicas da microbiota cutânea de indivíduos de A. leucopygius. Dentre os microorganismos encontrados estão Staphylococcus aureus, Staphylococcus epidermidis, Streptococcus sp., além da presença de fungos da ordem Eurotiales.

Palavras-chave: Aplastodiscus leucopygius; Microbiota; Cutânea; Anuros.

\footnotetext{
${ }^{1}$ Faculdade de Educação e Artes/Universidade do Vale do Paraíba, Brasil. E-mail: nolasco.laryssa@gmail.com.

2 Faculdade de Educação e Artes/Universidade do Vale do Paraíba, Brasil. E-mail: marindn@hotmail.com.

${ }^{3}$ Faculdade de Educação e Artes/Universidade do Vale do Paraíba, Brasil. E-mail: cladineia_bio@hotmail.com.

${ }^{4}$ Faculdade de Educação e Artes/Universidade do Vale do Paraíba, Brasil. E-mail: jonatasafn@gmail.com.

${ }^{5}$ Faculdade de Educação e Artes/Universidade do Vale do Paraíba, Brasil. E-mail: mmoroti@gmail.com.

${ }^{6}$ Faculdade de Educação e Artes/Universidade do Vale do Paraíba, Brasil. E-mail: cpsoares@univap.br.

${ }^{7}$ Faculdade de Educação e Artes/Universidade do Vale do Paraíba, Brasil. E-mail: flavia@univap.br.
} 